\title{
A Preliminary Abundance Estimate of an Atlantic Sturgeon (Acipenser oxyrinchus oxyrinchus) Contingent Within an Open Riverine System
}

\author{
Austin J. Flanigan ${ }^{1,3^{*}}$, Noah G. Perlut ${ }^{1}$, and James A. Sulikowski ${ }^{1,2}$ \\ ${ }^{1}$ University of New England, School of Marine and Environmental Programs, \\ Biddeford, ME 04005 \\ ${ }^{2}$ New College of Interdisciplinary Arts \& Sciences, Arizona State University, \\ Glendale, AZ 85306 \\ ${ }^{3}$ College of Fisheries and Ocean Science, University of Alaska Fairbanks, \\ Fairbanks, AK 99709 \\ "Austin Flanigan, Email: aflanigan@alaska.edu, Phone: (207) 806-9009 \\ Noah Perlut, Email: nperlut@une.edu, Phone: (207) 602-2778 \\ James Sulikowski, Email: James.Sulikowski@asu.edu, Phone: (602) 543-1214, Fax: (602) 543-6073
}

Flanigan, A.J., Perlut, N.G., and Sulikowski, J.A. 2021. A Preliminary Abundance Estimate of an Atlantic Sturgeon (Acipenser oxyrinchus oxyrinchus) Contingent Within an Open Riverine System. J. Northw. Atl. Fish. Sci., 52: 39-47. https://doi.org/10.2960/J.v52.m726

\begin{abstract}
Abundance estimates are essential for fisheries management, but estimating the abundance of open populations with low recapture rates has historically been unreliable. However, by using mark-recapture data modulated with survivability parameters obtained from analysis of acoustic telemetry data, more accurate abundance estimates can be made for species that exhibit these characteristics. One such species is the Atlantic sturgeon, for which abundance estimates were designated a research priority following precipitous population declines throughout the $20^{\text {th }}$ century. We addressed this research need in the Saco River Estuary (SRE), a system where the Atlantic sturgeon has been extensively studied using mark-recapture and acoustic telemetry methods since 2009. These data were analyzed using Bayesian analysis of a Lincoln-Peterson estimator, constrained with parameters from a Cormack-JollySeber model, to provide an initial abundance estimate for the system. The resulting estimate indicated that approximately 3299 (95\% Credible Interval: 1 462-6 828) Atlantic sturgeon utilize the SRE yearly, suggesting that the SRE provides critical foraging habitat to a large contingent of the species within the Gulf of Maine. The present study demonstrated the method utilized herein was effective in generating a reasonable estimate of abundance in an open system where recapture events are rare, and therefore may provide a valuable technique for supplying initial estimates of fish abundance in additional systems that display similar characteristics.
\end{abstract}

Keywords: Atlantic sturgeon, Gulf of Maine, abundance, acoustic telemetry, mark-recapture

\section{Introduction}

Estimating fish abundance is a cornerstone of fisheries management, especially for those species that are listed under the Endangered Species Act. Abundance information is used for establishing management strategies, determining species status, and measuring population recovery (Couturier et al., 2013). In the absence of abundance estimates, managers cannot effectively assess and implement stock management strategies, which may lead to further degradation of a stock. Despite the importance of quantifying abundance, this has not been accomplished for many fish stocks, particularly for those that exhibit open populations: The presence of both emigration and immi- gration within open populations violates the assumptions of traditional mark-recapture estimation techniques, which require closed systems (Seber, 1986). When utilized, these traditional methods yield highly variable and imprecise predictions (Kendall, 1999). As a result, some past studies have estimated the abundance of open populations by analyzing aspects of the species life history where the population displays closed behavior. For example, salmon population studies have performed mark-recapture techniques on salmon parr, when the juvenile fish are unable to leave their natal estuaries (Rodgers et al., 1992). Similarly, the Hudson River Atlantic sturgeon (Acipenser oxyrinchus oxyrinchus) population was estimated using juveniles younger than two years of age, ensuring that 
fish were confined to the river system (Peterson et al., 2000). This approach is not always possible, and as such, additional methods for estimating open population abundance are needed.

In recent years, several new techniques have been established for estimating abundance with mark-recapture data within open systems, including various parameterizations of the Jolly-Seber model (JS). These models account for fish movements in and out of the system by estimating catchability parameters, such as apparent survival and return probability (Seber, 1986). However, mark-recapture sampling procedures often violate model assumptions, resulting in biases that cause these models to generate errant abundance estimates (Carothers, 1973). The Cormack-Jolly-Seber (CJS) model was formulated to accurately estimate catchability parameters through the inclusion of external covariates (Lebreton et al., 1992), but this model lacks the ability to provide an abundance estimate. As a result, studies began utilizing acoustic encounter histories to better approximate these catchability parameters, which are then treated as fixed and incorporated into Jolly-Seber models, such as the POPAN formulation, and used to estimate abundance (Withers et al., 2019). Furthermore, Bayesian estimation techniques have been employed to reduce model uncertainty by incorporating prior knowledge into abundance estimates (Dudgeon et al., 2015; Ketz et al., 2018). Despite these advances in modeling procedures, these techniques still suffer from significant shortcomings, primarily that they are computationally difficult and often suffer from inflation bias due to low recapture numbers (Carothers, 1973; Cowen and Schwarz, 2006; Haxton and Friday, 2019). As a result, abundance estimation for open populations with rare recapture events remains difficult. The Atlantic sturgeon is one species that exhibits these characteristics and currently lacks abundance estimates.

The Atlantic sturgeon is a large, long lived, mobile, anadromous fish species inhabiting coastal waters and estuaries along the eastern seaboard of North America (NOAA, 2019). Due to its size and accessibility, this species faced intense fishing pressure that resulted in precipitous population declines throughout the $20^{\text {th }}$ century (Altenritter et al., 2017). These population declines led to both a moratorium being placed on all harvest in 1998 (ASMFC, 2021) and the species being listed under the United States Endangered Species Act in 2012. Here, Atlantic sturgeon were separated into five distinct population segments (DPS), with the Gulf of Maine (GOM) DPS listed as Threatened, and all others as Endangered (NOAA, 2019). As such, abundance estimates are a research priority for the species (ASSRT, 2007); however, the aforementioned shortcomings in abundance estimation methods have limited our understudying of this ecological parameter for Atlantic sturgeon (Hilton et al., 2016), as the species exhibits open populations. Atlantic sturgeon sub-adult and adult individuals are highly migratory, traveling long distances between multiple river systems during summer months and wintering in coastal marine waters (Altenritter et al., 2017). Despite these movement patterns, Atlantic sturgeon are known to exhibit fidelity to both foraging grounds and natal spawning rivers (Fernandes et al., 2010). Since this species regularly returns to specific estuaries over time, their abundance will vary across river systems, and therefore the number of Atlantic sturgeon must be estimated for each respective river system (Wirgin et al., 2018).

The Saco River Estuary (SRE) in the GOM is a unique system for Atlantic sturgeon. After extirpation in the 1960 s, they were discovered to have returned to the river system in 2007 (Furey and Sulikowski, 2011). Since this re-emergence, the fish have been extensively studied using both mark-recapture and acoustic telemetry methods. These efforts have shown Atlantic sturgeon are unable to spawn in the SRE, and instead are using the system as a foraging ground (Novak et al., 2017). The primary use of the SRE as a foraging habitat indicates that all individuals using the SRE are adults and sub-adults from other natal estuaries in the GOM DPS, resulting in an open and highly variable contingent comprised of multiple spawning populations (Wippelhauser et al., 2017). The nature of this system and the extensive dataset available provides an opportunity to estimate how many Atlantic sturgeon use the SRE as a foraging ground. Given this opportunity, the goals of this study were (1) to develop an appropriate method for approximating fish abundance using a synthesis of mark-recapture, acoustic telemetry, and Bayesian estimation techniques, and (2) to apply this method to Atlantic sturgeon within the SRE in order to estimate the number of individuals utilizing the river system.

\section{Methods}

This study was a component of a larger investigation of Atlantic sturgeon within the SRE, which was comprised of long-term acoustic monitoring and mark-recapture sampling spanning 2009-2018. As a result, capture and tagging efforts varied over time, particularly during initial years of the study. As such, standardized efforts from 2014-2018 will be detailed here.

\section{Fish Capture}

Atlantic sturgeon were captured and sampled from midMay through mid-November each year, with a target frequency of one sampling attempt per week. Given 
seasonal variability, this did not always occur during the spring and late autumn, where the majority of sampling opportunities occurred during the summer months. Fish were captured using gillnets (91.4 m long, $2 \mathrm{~m}$ high), which were composed of either 15.24 or $30.48 \mathrm{~cm}$ bar mesh. These nets were placed between the jetties at the mouth of the Saco River, where they were deployed for a standard of 15 minutes, as longer net soaks yielded too many individuals. The nets were hand hauled, and all entangled sturgeon were extracted and brought onto the deck of the boat. Sturgeon were then transported back to the University of New England dock, where they were washed with estuarine water to oxygenate their gills during the five minute steam. At the dock, fish were placed into net pens $(2.1 \times 0.9 \times 0.9 \mathrm{~m})$ for a recovery period ( $\sim 15 \mathrm{~min}$ ) before undergoing research and handling protocols outlined by Kahn and Mohead (2010). The protocols constituted measuring fork length to the nearest $\mathrm{mm}$, visually searching for external tags, and scanning (AVID PowerTracker VIII) for internal PIT tags. If no tags were detected, a 134.2-kHz PIT tag (model HPT12, Biomark) was inserted adjacent to the dorsal fin. As a secondary means of identification, we inserted a spaghetti T-bar tag on the opposing side of the dorsal fin. Following tagging procedures, fish were then released back into the river.

In addition to these traditional tagging efforts, a subsample of fish was also affixed with acoustic transmitters following the methods in Novak et al. (2017). These individuals were selected to reflect the size range of all captured individuals, but only those deemed to be in the best condition, or lacking physical injury, were selected to be acoustically tagged. We surgically implanted an acoustic transmitter (model V16; $69 \mathrm{KHz}, 16 \mathrm{~mm}$ diameter, approximate 2 500-d battery life; VEMCO, Halifax, Nova Scotia) into the abdominal cavity of these fish. A 5-cm incision was made on the midline of the body on the ventral surface, where the transmitter was anteriorly inserted after being coated in antibiotic ointment. The incision was closed using one or two polydioxanone sutures (PDO II violet monofilament absorbable suture; Oasis, Mettawa, Illinois), which was then coated in additional antibiotic ointment. The surgical process lasted approximately 10 minutes, and fish were then returned to the net pen for a recovery period prior to release.

\section{Acoustic Monitoring}

Following the methods of Novak et al. (2017), we deployed an acoustic array within the SRE seasonally from 2009-2018. A total of seven acoustic receivers (model VR2W; VEMCO, Halifax, Nova Scotia) were distributed from the mouth of the Saco River to the Cataract dam at river $\mathrm{km} 10$ (Fig. 1), where their placement served to maximize the area where tagged fish would be detectable within the SRE. These receivers were deployed each year during the month of May and were removed from the river in late November or early December. During this deployment period, receivers were downloaded once per month; any required maintenance was performed during these data collection periods. Additionally, during the winter months, two receivers were placed at the river mouth. These receivers remained deployed from the removal of the acoustic array in early winter until its re-deployment in the spring. This ensured that throughout the entire year no acoustically tagged fish could enter or leave the river system without being detected by an acoustic receiver.

From the acoustic data, we created a binary annual encounter history for the presence-absence of acoustically tagged fish for each year the acoustic array was deployed. The acoustic data were also used to calculate Atlantic sturgeon residence time, $T_{\text {res }}$, within the SRE. For all acoustically tagged fish that were found to have returned in 2017, the year with the largest number of active acoustic transmitters, we tallied the number of days that each fish spent within the river system: The median number of days present across all individuals provided $T_{r e s}$. Finally, we created a distribution of the number of distinct acoustically tagged Atlantic sturgeon detected per month, ranging from May to November. Here, the total detections from 2017 were used, as this was the year with the largest number of transmittered fish returning $(n=36)$. These detections were filtered by month to contain only unique fish detections, which were then used to calculate the proportion of total yearly unique detections, and hence represent the proportion of the total population present each month.

\section{Data Analysis}

Catchability Parameters: The annual acoustic encounter history was analyzed using a Cormack-Jolly-Seber (CJS) model in program MARK through the RMark interface (RMark, 2013). This analysis estimates both the probability of apparent annual survival $(\Phi)$ and the probability of return $(p)$ of an open population (Lebreton et al., 1992). Given that acoustic tag batteries only lasted seven years, and that this study covers a 10-year time period, some tags were known to have expired during the study. As such, these tags were removed from the analysis following expiration. The candidate model set included all combinations, where the estimated $\Phi$ and $p$ parameters were either constant (c) or time dependent (t) (Perlut and Strong, 2016), and the individual covariate of fork length (fl) was included in estimating $\Phi$. Program MARK applies an information theoretic approach, where all models 


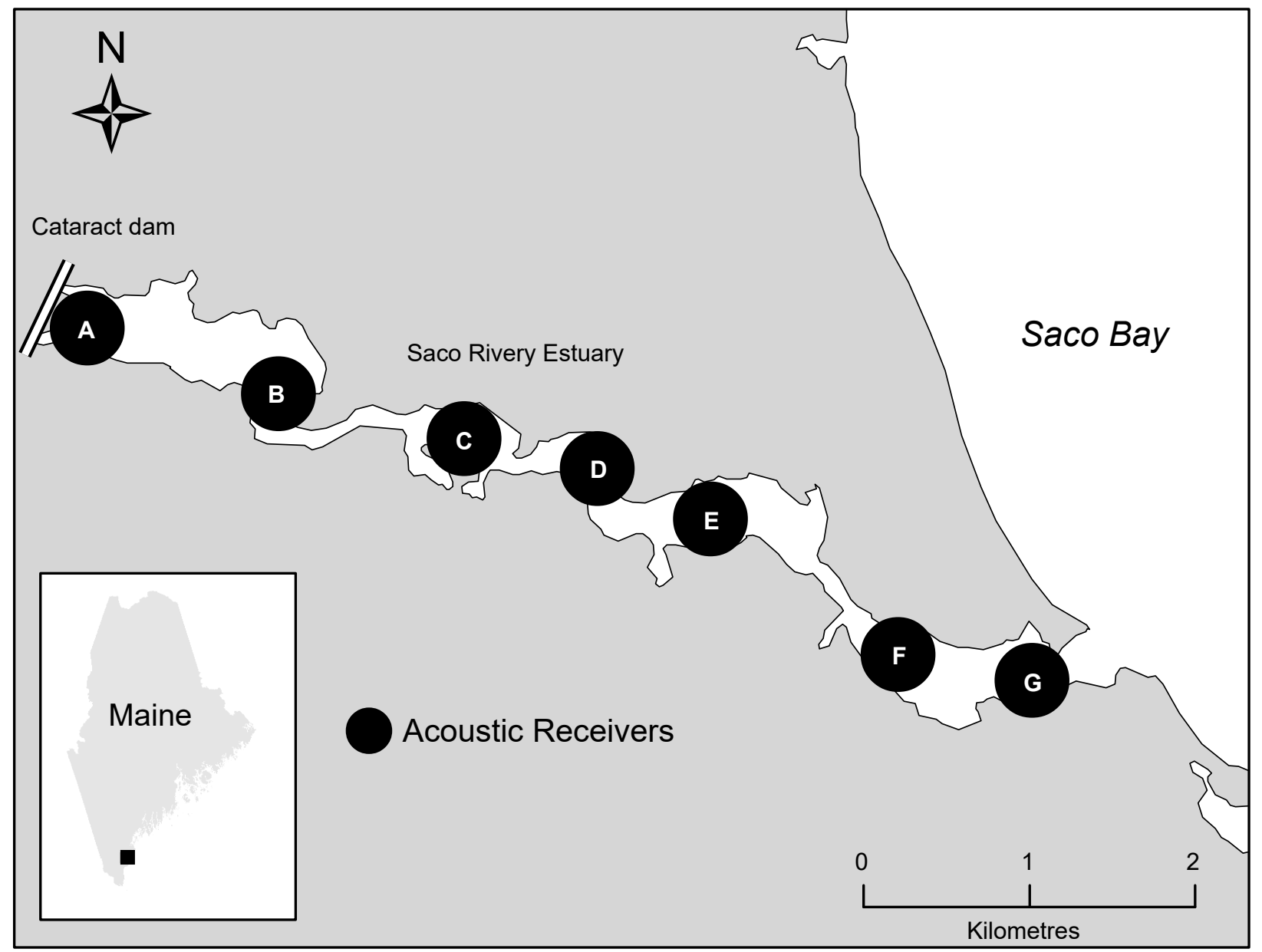

Fig. 1: Map of the Saco River Estuary, where each of the seven acoustic receivers' locations and approximate detection range is represented by black circles. Receivers were placed from the Cataract Dam (A) to the University of New England Marine Science Center beach $(\mathbf{G})$.

with $\triangle \mathrm{AIC}<2.0$ are considered biologically relevant models in explaining variation in the dataset. Biologically significant covariates within these top ranking models were identified as those whose $95 \%$ confidence interval of the beta estimates did not include zero (Burnham and Anderson, 2002).

The estimates for the $\Phi$ and $p$ parameters obtained from the acoustic encounter history were then treated as fixed parameters (Withers et al., 2019) and used to determine the number of catchable tagged fish each year $\left(C_{y}\right)$ via equation 1:

$$
C_{y}=\sum_{\mathrm{t}=2009}^{\mathrm{y}-1}\left(m_{t} \times \prod_{j=t+1}^{y}\left(\Phi_{\mathrm{j}} \times \mathrm{p}_{\mathrm{j}}\right)\right)
$$

The number of fish tagged, or marked, in a study year $\left(m_{t}\right)$ was multiplied by the product of the probability that those fish survived and returned each of the following study years $\left(\Phi_{\mathrm{j}} \times \mathrm{p}_{\mathrm{j}}\right)$, up to year $\mathrm{y}$, yielding the number of
Atlantic sturgeon tagged in year $t$ that return to the system in year $\mathrm{y}$. Then, by summing across all Atlantic sturgeon tagged in study years prior to year $y$, we calculated the total number of catchable tagged fish in year $y$. Here, $t$ ranged from 2009 to 2017 and y from 2010 to 2018 .

Abundance Estimate: In order to estimate abundance for each year, we used the Lincoln-Peterson (Seber, 1986) mark-recapture model. This estimator states the ratio of captured marked to unmarked fish is equivalent to the ratio of all previously marked fish to total fish abundance; however, this model is traditionally used to estimate closed populations, requiring the model to be reworked in order to estimate the number of Atlantic sturgeon utilizing the SRE, an open system where fish are not always catchable. As a result, the model was modified to estimate abundance annually by using $C_{y}$. Annual abundance, $N_{y}$, can be represented as: 
$N_{y}=\frac{C y}{r_{y} / m_{y}}$

Here, annual abundance can be estimated by dividing the catchable tagged population in a given year by the ratio of marked $\left(r_{y}\right)$ to unmarked $\left(m_{y}\right)$ capture events in that same year. Uncertainty in this approach can be mitigated by incorporating prior knowledge (Madigan and York, 1995) and recasting the Lincoln-Peterson estimator in a Bayesian framework. We accomplished this by representing the ratio of marked to unmarked capture events as the probability that a captured fish is marked, or $\mathrm{p}(\mathrm{t})_{\mathrm{y}}$. Each year can then be considered an individual mark-recapture experiment, with unique yearly values of $\mathrm{p}(\mathrm{t})_{\mathrm{y}}$. This parameter was then estimated separately for each year $y$ using the rjags package in R (Plummer, 2019). A binomial likelihood was used, where:

$\mathrm{L}\left(p(t)_{y} \mid r_{y}, m_{y}\right)=\left(\begin{array}{l}m_{y} \\ r_{y}\end{array}\right) \times p(t)_{y}^{r_{y}}\left(1-p(t)_{y}\right)^{m_{y}-r_{y}}$

Three separate beta priors were then utilized, with parameters $(1,1),(6,54)$, and $(9,81)$. These three parameterizations represent a noninformative (uniform prior) and two informative priors with the probability that a given fish is tagged centered around 0.1 . The informative priors represent prior estimates, with $95 \%$ confidence, that $\mathrm{p}(\mathrm{t})_{\mathrm{y}}$ is between $(0.038,0.187)$ and $(0.047,0.169)$, respectively. As the experts, these priors were selected to represent a plausible range of $\mathrm{p}(\mathrm{t})_{\mathrm{y}}$ values: This included the exclusion of extremely low $\mathrm{p}(\mathrm{t})_{\mathrm{y}}$ values, where low numbers of recapture events, and correspondingly low recapture probabilities, can lead to inflation bias in abundance estimation (Haxton and Friday, 2018). Informative prior selection then served to prevent inflation bias by constraining the upper bound on the abundance estimate, therefore providing a more conservative estimate of the contingent size.

We ran Markov Chain Monte Carlo estimations for each of these priors, with an adaptive phase of 1000 iterations, followed by 10000 iterations of draws from the posterior distribution that were summarized to estimate the probability of a captured fish being tagged. Model performance was evaluated with both density and trace plots for each of the Markov chains. The annual mean and 95\% credible set abundance estimates were then obtained using the yearly mean and $95 \%$ credible set estimates of $\mathrm{p}(\mathrm{t})_{\mathrm{y}}$. Following this, every draw for each $\mathrm{p}(\mathrm{t})_{\mathrm{y}}$ posterior distribution was used to calculate an annual abundance estimate, generating posterior distributions of abundance estimates in each year. These posterior distributions were then pooled together, with the final estimate of abundance and the $95 \%$ credible set being taken as the mean and $95 \%$ credible interval of the pooled distribution.

\section{Results}

Between 2009-18, a total of 762 Atlantic sturgeon were conventionally tagged in the SRE. These fish ranged in size from $65-199 \mathrm{~cm}$ in FL, with a mean \pm SD length of $127.5 \pm 23.4 \mathrm{~cm}$. Of these tagged fish, 30 were recaptured in subsequent years (Table 1). Additionally, 74 Atlantic sturgeon were implanted with acoustic transmitters, ranging from 77-190 $\mathrm{cm}$ in FL with a mean \pm SD 137.1 \pm 23.7 $\mathrm{cm}$. In 2016-18, where sampling efforts were standardized, the catch per unit effort (CPUE) was 0.47 fish per minute in a $91.4 \mathrm{~m}$ net.

Table 1. Summary of all model parameters utilized in calculating Ny, with $\mathrm{p}(\mathrm{t}) \mathrm{y}$ representing the parameter estimates for the beta $(1,1)$, beta $(6,54)$, and beta $(9,81)$ priors, respectively.

\begin{tabular}{ccrrrrll}
\hline \hline YEAR & \multicolumn{1}{c}{$\Phi$} & \multicolumn{1}{c}{$\boldsymbol{P}$} & MT & MY & RY & CY & \multicolumn{1}{c}{ P(T)Y } \\
\hline $\mathbf{2 0 0 9}$ & 0.959 & 1 & 33 & 33 & NA & NA & NA \\
$\mathbf{2 0 1 0}$ & 0.959 & 1 & 54 & 55 & NA & NA & NA \\
$\mathbf{2 0 1 1}$ & 0.959 & 0.898 & 96 & 96 & NA & NA & NA \\
$\mathbf{2 0 1 2}$ & 0.959 & 0.903 & 60 & 64 & 4 & 155 & $(0.072,0.079,0.083)$ \\
$\mathbf{2 0 1 3}$ & 0.959 & 1 & 100 & 103 & 3 & 186 & $(0.038,0.055,0.062)$ \\
$\mathbf{2 0 1 4}$ & 0.959 & 0.774 & 132 & 136 & 4 & 274 & $(0.036,0.050,0.057)$ \\
$\mathbf{2 0 1 5}$ & 0.959 & 0.64 & 60 & 64 & 4 & 300 & $(0.076,0.081,0.085)$ \\
$\mathbf{2 0 1 6}$ & 0.959 & 0.82 & 80 & 82 & 2 & 222 & $(0.035,0.056,0.064)$ \\
$\mathbf{2 0 1 7}$ & 0.959 & 0.619 & 58 & 64 & 6 & 237 & $(0.107,0.096,0.098)$ \\
$\mathbf{2 0 1 8}$ & NA & NA & 98 & 104 & 6 & 175 & $(0.066,0.073,0.077)$ \\
\hline
\end{tabular}


During the 2017 season, where the acoustic array was deployed for 180 days, the median residency time $\left(T_{\text {res }}\right)$ was 15.5 days. The monthly distribution of the percent of the population available for capture indicated sturgeon abundance peaked from July to September, with roughly $20 \%$ of the contingent present during each of these three months (Fig. 2).

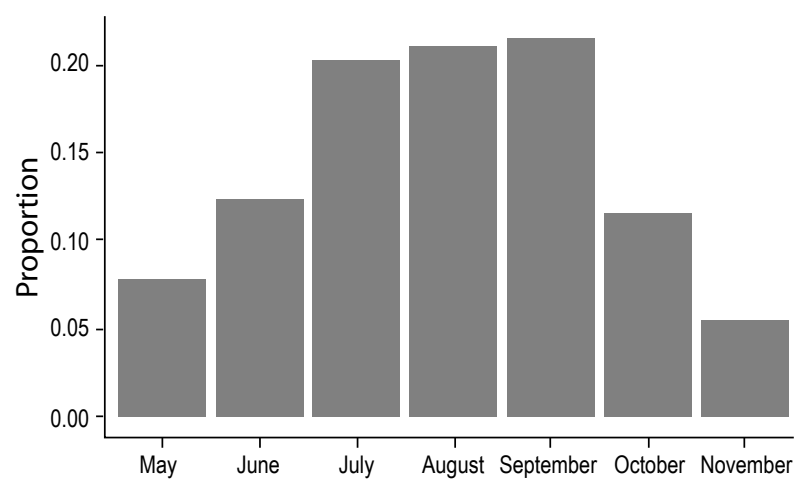

Fig. 2: The proportion of the SRE Atlantic Sturgeon contingent present by month. While sturgeon are present for a total of six months each year, abundance peaks from July to September.

The top ranked CJS survival model was $\Phi_{\mathrm{c}} p_{\mathrm{t}}$, which explained $\sim 85 \%$ of the variation in the detection data. The probability of apparent survival $\Phi$ was constant, while the probability of return $p$ was time dependent: Fork length was not included in the final model. The resulting apparent survival estimate was 0.959 , and the annual detection probability estimate varied from 0.619 to 1 throughout the study. These parameter estimates were then used to calculate the catchable tagged population for each year from 2012 to 2018. Abundance estimation was not conducted until 2012 due to the small number of individuals tagged in years prior (2009-2011). The annual $\mathrm{p}(\mathrm{t})_{\mathrm{y}}$ estimates from the Bayesian analysis with the noninformative and two informative priors ranged from 0.035 to 0.107 , with trace and density plots indicating clear model convergence across study years and priors. Values for $\mathrm{p}(\mathrm{t})_{\mathrm{y}}$ and all other abundance estimation parameters are summarized in Table 1.

The constrained Lincoln-Peterson model, for each of the three priors, yielded overall abundance estimates of 5492 (95\% Credible Interval: 1 374-17 989), 3693 (95\% Credible Interval: 1 476-8 436), and 3299 (95\% Credible Interval: 1 462-6 828) sturgeon that utilize the SRE over the course of the year. Estimates of abundance for each year across the three priors are described in Table 2.

\section{Discussion}

While many current abundance estimation methods struggle to generate reasonable estimates of abundance for open populations, where recapture events are rare, the present study established an alternate approach that effectively approximated the number of Atlantic sturgeon utilizing the SRE. Using the most informative prior (Beta $(9,54)$, the initial application of this method suggested that the SRE provides foraging habitat (Novak et al., 2017) to approximately 3299 Atlantic sturgeon each year. The mean estimates from all three priors were remarkably similar, indicating that results were primarily driven by the data likelihood; however, the incorporation of prior knowledge using the informative priors constrained the $95 \%$ credibility interval by significantly lowering the upper-bound, providing a more conservative credible set for the contingent abundance estimate. Furthermore, the lower bound on the population estimate was robust, as for all priors used, the data strongly suggested that the SRE is utilized by approximately 1400 Atlantic sturgeon each year.

In addition to the abundance estimate, the CJS analysis also performed well, where the top ranked model explained $85 \%$ of acoustic data variability and provided reasonable estimates of both apparent survival and return probability. The present study found a remarkably high rate of annual apparent survival, $95.9 \%$, which is consistent with estimates from previous acoustic telemetry studies of sturgeon populations. For example, Withers et al., (2019) found survival rates of $94.6 \%$ for lake sturgeon (Acipenser fulvescens) in Lake Erie, while Hightower et al., (2016) found a survival rate of $86.0 \%$ for Atlantic sturgeon in the Carolina DPS. Additionally, while all sturgeon emigrated from the SRE each winter, the CJS return rates suggested that $62-100 \%$ of surviving sturgeon would return to the estuary in the following year. This finding indicates high site fidelity and long-term usage of the SRE within the Atlantic sturgeon GOM population, as well as confirms findings of past studies, which found return rates of $69 \%$ (Wippelhauser et al., 2017). Further, these annual return rates are similar to those of Atlantic sturgeon from the Penobscot River (up to 95\%), a nearby system deemed an important estuary for the GOM Atlantic sturgeon DPS (Altenritter et al., 2017). As a result of this designation and the abundance estimate herein, the present study suggests the SRE is also a river system critical to the Atlantic sturgeon GOM DPS.

The SRE is a relatively small estuarine system, and so the estimated abundance may appear disproportionately high, but our abundance estimate is supported by other data. For 
Table 2. Yearly sturgeon abundance estimates $\left(\mathrm{N}_{\mathrm{y}}\right)$, and $95 \%$ credible intervals, using each of the three priors.

\begin{tabular}{crrrrrr}
\hline \hline YEAR & \multicolumn{2}{c}{ BETA(1,1) } & \multicolumn{2}{c}{ BETA(6, 54) } & \multicolumn{2}{c}{ BETA(9, 81) } \\
\hline $\mathbf{2 0 1 2}$ & 2152 & $(1078-6660)$ & 1973 & $(1111-4008)$ & 1876 & $(1172-3456)$ \\
$\mathbf{2 0 1 3}$ & 4902 & $(2250-17929)$ & 3367 & $(1971-7280)$ & 2997 & $(1864-5641)$ \\
$\mathbf{2 0 1 4}$ & 7585 & $(3750-23170)$ & 5461 & $(3271-11084)$ & 4817 & $(3030-8764)$ \\
$\mathbf{2 0 1 5}$ & 3968 & $(2029-11719)$ & 3718 & $(2206-7593)$ & 3531 & $(2221-6517)$ \\
$\mathbf{2 0 1 6}$ & 6266 & $(2664-29934)$ & 3983 & $(2260-8993)$ & 3476 & $(2112-6873)$ \\
$\mathbf{2 0 1 7}$ & 2224 & $(1239-5363)$ & 2460 & $(1555-4590)$ & 2442 & $(1613-4277)$ \\
$\mathbf{2 0 1 8}$ & 2654 & $(1374-6479)$ & 2396 & $(1480-4654)$ & 2276 & $(1492-3921)$ \\
\hline
\end{tabular}

example, the standardized CPUE from 2016- 2018 was 0.31 Atlantic sturgeon per hour per metre of net soaked. A similar study on the Penobscot River had an average annual CPUE of only 0.016 sturgeon per hour per metre of net (Altenritter et al., 2017), and while this is a much larger system, both density and catch rate of Atlantic sturgeon in the SRE are higher. Further, not all the fish are utilizing the river system concurrently. A median residency time of 15.5 days suggests high turnover rates within the system, and the proportion of the contingent present within a given month never exceeds $22 \%$. With acoustic data and sampling indicating sturgeon are prevalent within the river from mid-May through mid-November (Novak et al., 2017), the aforementioned data suggests approximately 330 Atlantic sturgeon are within the river during the months of July, August, and September, when abundance is highest. Conversely, in the late spring and autumn months, closer to 165 fish are within the river system at any given time. In all, the abundance estimates from this study indicate that the SRE is supporting a large, but highly variable, contingent of Atlantic sturgeon each year.

Despite the ability to derive both reasonable population estimates and catchability parameters within the SRE, there are caveats to this approach. The current study used existing software in a two-step approach, providing a mathematically simplistic and user-friendly model; however, this required CJS parameter estimates from the first step to be treated as fixed parameters when estimating abundance (Dudgeon et al., 2015), and therefore uncertainty in these parameters was not incorporated into the final estimate of abundance. In addition, we assumed that contingent size was constant across years, and as such, the final estimate of abundance was taken as the mean from a pooled distribution across years. Violation of this assumption would require independent abundance estimates for each study year, but abnormally low numbers of recapture events across years may lead to high levels of uncertainty and inflated abundance estimates (Withers et al., 2019). Bayesian estimation can mitigate this uncertainty by using informative priors to constrain the credible set (Madigan and York, 1995), but increased recapture rates are needed to provide more precise abundance estimates. It is therefore recommended that the population estimates produced by the methods herein are used as approximate estimates for the magnitude of abundance, and that these estimates are only generated when studies have many years of mark-recapture data.

Given the assumptions of our approach, additional efforts are needed to improve and refine this method of abundance estimation. An integrated Bayesian modeling approach would better quantify model uncertainty, but this requires the CJS model to be coded into the rjags package (Dudgeon et al., 2015). Additionally, shortcomings in data collection must be addressed in order to improve model performance: Low recapture rates continue to restrict mark-recapture abundance estimation effectiveness, with large amounts of long-term data being required for estimates to be reliable (Haxton and Friday, 2018; Withers et al., 2019). Until further model development occurs, our approach provides a useful method for generating preliminary estimates of fish abundance in numerous systems where this has historically been unfeasible.

\section{Acknowledgments}

We thank the undergraduate and graduate students of the Sulikowski laboratory, as well as the Marine Science Center staff at the University of New England for logistical support. We also thank Dr. Margaret Short for her assistance with Bayesian estimation methods. This research was approved by UNE's Animal Care and Use Committee (IACUC protocol no.20101221SUL, UNE20140401SULIJ, and 033117-001). 


\section{References}

Altenritter, M. N., Zydlewski, G., Kinnison, M. T., and Wippelhauser, G. S. 2017. Atlantic sturgeon use of the Penobscot River and marine movements within and beyond the Gulf of Maine. Marine and Coastal Fisheries Dynamics Management and Ecosystem Science. 9(1): 216-230. https://doi.org/10.1080/19425120.2017.1282898

ASMFC (Atlantic States Marine Fisheries Commission). 2021. Atlantic Sturgeon [Internet]. [Cited 2021 May 15]. Available from: http://www.asmfc.org/species/atlanticsturgeon

ASSRT (Atlantic Sturgeon Status Review Team). 2007. Status review of Atlantic Sturgeon (Acipenser oxyrinchus oxyrinchus). Report to the National Marine Fisheries Service, Gloucester, Massachusetts.

Burnham, K. P., and Anderson, D. R. 2002. Model selection and multimodel inference, second edition. Springer, New York, New York, USA.

Carothers, A. D. 1973. The effects of unequal catchability on Jolly-Seber estimates. Biometrics. 29(1): 79-100. https:// doi.org/10.2307/2529678

Couturier, T., Cheylan, M., Bertolero, A., Astruc, G., and Besnard, A. 2013. Estimating abundance and population trends when detection is low and highly variable: A comparison of three methods for the Hermann's tortoise. Wildlife Management. 77(3): 454-462. https://doi. org/10.1002/jwmg.499

Cowen, L., and Schwarz, C. J. 2006. The Jolly-Seber model with tag loss. Biometrics. 62(3): 699-705. https://doi. org/10.1111/j.1541-0420.2006.00523.x

Dudgeon, C. L., Pollock, K. H., and Braccini, J. M. 2015. Integrating acoustic telemetry into mark-recapture models to improve the precision of apparent survival and abundance estimates. Oecologia, 178: 761-772. https://doi. org/10.1007/s00442-015-3280-Z

Fernandes, S. J., Zydlewski, G. B., Zydlewski, J. D., Wippelhauser, G. S., and Kinnison, M. T. 2010. Seasonal distribution and movements of Shortnose Sturgeon and Atlantic Sturgeon in the Penobscot River Estuary, Maine. Transactions of the American Fisheries Society. 139: 1436-1449. https://doi.org/10.1577/T09-122.1

Furey, N. B., and Sulikowski, J. A. 2011. The fish assemblage structure of the Saco River Estuary. Northeast Nature. 18: 37-44. https://doi.org/10.1656/045.018.0104

Haxton, T. J., and Friday, M. J. 2019. Are we overestimating recovery of sturgeon populations using mark/recapture surveys? Journal of Applied Ichthyology, 35: 336-343. https://doi.org/10.1111/jai.13795

Hightower, J. E., Loeffler, M., Post, W. C., and Peterson, D. L. 2015. Estimated survival of subadult and adult Atlantic sturgeon in four river basins in the Southeastern United States. Marine and Coastal Fisheries. 7(1): 514-522. https://doi.org/10.1080/19425120.2015.1088491

Hilton, E. J., Kynard, B., Balazik, M. T., Horodysky, and A. Z., Dillman, C. B. 2016. Review of the biology, fisheries, and conservation status of the Atlantic Sturgeon, Acipenser oxyrinchus oxyrinchus Mitchill, 1815. Journal of Applied
Ichthyology, 32: 30-66. https://doi.org/10.1111/jai.13242

Kahn, J., and Mohead, M. 2010. A Protocol for Use of Shortnose, Atlantic, Gulf, and Green Sturgeons. NOAA Technical Memorandum. NMFS-OPR-45.

Kendall, W. L. 1999. Robustness of closed capture-recapture methods to violations of the closure assumption. Ecology. 80(8): 2517-2525. https://doi.org/10.2307/177237. https:// doi.org/10.1890/0012-9658(1999)080[2517:ROCCRM] 2.0.CO;2

Ketz, A. C., Johnson, T. L., Monello, R. J., Mack, J. A., George, J. L., Kraft, B. R., Wild, M. A., Hooten, M. B., and Hobbs, N. T. 2018. Estimating abundance of an open population with an $\mathrm{N}$-mixture model using auxiliary data on animal movements. Ecological Applications, 28: 816-825. https:// doi.org/10.1002/eap.1692

Lebreton, J. D., Burnham, K. P., Clobert, J., and Anderson, D. R. 1992. Modeling survival and testing biological hypotheses using marked animals: a unified approach with case studies. Ecological Monographs, 62: 67-118. https:// doi.org/10.2307/2937171

Madigan, D., York, J. 1995. Bayesian graphical models for discrete data. International Statistical Review, 6(2): 215-232. https://doi.org/10.2307/1403615

Novak, A. J., Carlson, A. E., Wipplehauser, G., Zydlewski, G., Kinnison, M. T., and Sulikowski, J. A. 2017. Critical foraging habitat of Atlantic Sturgeon (Acipenser oxyrinchus oxyrinchus) based on feeding habits, prey distribution, and movement patterns in the Saco River estuary, Maine. Transactions of the American Fisheries Society, 146(2): 308-317. https://doi.org/10.1080/00028487.2016.1264472

NOAA Fisheries. 2019. Atlantic Sturgeon (Acipenser oxyrinchus oxyrinchus) [Internet]. [Cited 2019 April 20]. Available from: http://www.fisheries.noaa.gov/pr/species/fish/ atlanticsturgeon.html.

Perlut, N. G., and Strong, A. M. 2016. Comparative analysis of factors associated with first-year survival in two species of migratory songbirds. Journal of Avian Biology. 47: 1-7. https://doi.org/10.1111/jav.00892

Peterson, D. L., Bain, M. B., and Haley, N. 2000. Evidence of declining recruitment of Atlantic sturgeon in the HudsonRiver. North American Journal of Fisheries Management, 20: 231-238. https://doi.org/10.1577/15488675(2000)020\%3C0231:EODROA\%3E2.0.CO;2

Plummer, M. 2019. rjags: Bayesian graphical models using MCMC. R package version 4-10. https://cran.r-project.org/ web/packages/rjags/index.html.

R Core Team, 2017. R: A language and environment for statistical computing. R Foundation for Statistical Computing, Vienna, Austria. https://www.R-project.org/.

RMark: "An R interface for analysis of capture-recapture data with MARK." AFSC Processed Rep. 2013-01, Alaska Fisheries Science Center, NOAA, National Marine Fisheries Service, Seattle, WA. http://www.afsc.noaa.gov/ Publications/ProcRpt/PR2013-01.pdf.

R Studio Team 2016. RStudio: Integrated development for R. RStudio, Inc., Boston, MA URL http://www.rstudio.com/.

Rodgers, J. D., Solazzi, M. F., Johnson, S. L., and Buckman, M. A. 1992. Comparison of three techniques to estimate 
juvenile Coho salmon populations in small streams. North American Journal of Fisheries Management, 12(1): 79-86. https://doi.org/10.1577/1548-8675(1992)012\%3C0079:CO TTTE\%3E2.3.CO;2

Seber, G. A. 1986. A review of estimating animal abundance. Biometrics. 42: 267-292. https://doi.org/10.2307/2531049

Wirgin, I., Roy, N. K., Maceda, L., and Mattson, M. T. 2018. DPS and population origin of subadult Atlantic sturgeon in the Hudson River. Fisheries Research. 207: 165-170. https://doi.org/10.1016/j.fishres.2018.06.004

Whippelhauser, G. S., Sulikowski, J., Zydlewski, G. B., Altenritter, M. A., Kieffer, M., and Kinnison, M. T. 2017.
Movements of Atlantic Sturgeon of the Gulf of Maine inside and outside of the geographically defined distinct population segment. Marine and Coastal Fisheries: Dynamics, Management, and Ecosystem Science: 9: 93-107. https://doi.org/10.1080/19425120.2016.1271845 Withers. J. L., Einhouse, D., Clancy, M., Davis, L., Neuenhoff, R., and Sweka, J. 2019. Integrating acoustic telemetry into a mark-recapture model to improve catchability parameters and abundance estimates of Lake sturgeon in Eastern Lake Erie. North American Journal of Fisheries Management, 39(5): 913-920. https://doi.org/10.1002/ $\underline{\text { nafm. } 10321}$ 